\title{
"Narraciones cotidianas", una experiencia de audiencia con niños/as de 1 a 2 años
}

Pág. 224- 241

*María Gabriela

Carrizo Méndez

**Stephanía

Cifuentes Oyarzún

$* *$ Daniela

Faúndez Silva

Educadoras Jardín Infantil KüpülweSantiago Chile

*mgcarrizo@uc.cl

**stefi.cifuentes@gmail.c om

dpfaundez@uc.cl

Fecha de Entrega: agosto de 2019.

Fecha de Aceptación: octubre de 2019.

\section{Resumen}

La educación inicial, cada vez más, se ha ido transformando para posicionar a los niños y niñas en el centro de la práctica educativa. Al observar con detención la infancia, sabemos que el interés por la narración oral, la búsqueda de la belleza, el arte y la sensorialidad, son parte intrínseca del desarrollo en los primeros años de vida.

La experiencia que se detalla, consiste en un proyecto de formación de audiencia infantil aplicado a un grupo de 13 niños, de entre 1 a 2 años, de un jardín infantil inspirado en la filosofía Reggio Emilia, ubicado en la ciudad de Santiago de Chile.

Este proyecto se desarrolló a nivel transversal en el aula, implementando diversas experiencias de narración, utilizando estrategias orales, corporales, sonoras y visuales. Los procesos vividos, desde la observación y el actuar de los niños, permitieron repensar una propuesta de formación de audiencia infantil para los primeros años, con nuevas prácticas, que pudiesen ahondar en las capacidades de los niños para vincularse con las propuestas educativas, así como sus posibilidades de acción sobre lo que nos parece tan lejano como la cultura y la comunidad.

\section{Palabras clave}

Primera infancia, formación de audiencia infantil, sensorialidad y arte, percepción, apreciación estética. 


\title{
"Everyday stories", an audience experience with 1 to 2 year-old children
}

\begin{abstract}
Early childhood education, increasingly, has been transforming to position children at the center of the educational practice. When we look closely at childhood, we know that interest towards oral narratives, the search of beauty, art and sensoriality, are intrinsic to development in the early years.
\end{abstract}

The experience detailed, consists of a project of audience formation in early childhood applied with a group of 13 one to two-year-old children, in an educational center inspired by the Reggio Emilia philosophy, at Santiago de Chile.

This project was developed transversally in the classroom, implementing diverse experiences of narration, using oral, bodily, sound and visual strategies. Processes lived, based on the observation and actions of the children, allowed us to rethink a proposal of audience formation in early childhood, with new practices that might delve into the children's capabilities to bond with the educational proposals, as well as their possibilities of action on something that seems to us so remote as is culture and community.

\section{Key words}

Early childhood, audience formation in early childhood, sensoriality and art, perception, aesthetic appreciation. 
"Narraciones cotidianas", una experiencia de audiencia con niños/as de 1 a 2 años

\section{Introducción}

Educar en primera infancia ha resignificado cada vez más aquello que los niños pueden vivir desde edades tempranas. Las nuevas miradas sobre el valor de la educación inicial han conllevado nuevas reformas en torno a lo que significa la educación parvularia en Chile, ante lo que urge, buscar nuevas maneras de educar, en las cuales el niño y la niña (en lo sucesivo "niño") se transformen en el centro de toda práctica pedagógica, desde sus intereses y miradas, hasta su capacidad de intervenir sobre las experiencias y momentos en las que está presente.

Una de estas formas que ha presentado particular atención es la filosofía Reggio Emilia, en la cual se considera la observación como un acto de conocimiento y como un acto creativo que requiere la interpretación de los educadores (Red Territorial de Educación Infantil de Cataluña, 2011). Es en esta observación del mundo infantil, donde podemos escuchar y conocer a los niños y niñas, en todos los momentos del día, entendiendo que están hechos de cien lenguajes, es decir, tienen múltiples maneras de comprender lo que perciben del mundo y por otro lado, expresarlo al hacer su pensamiento visible (Edwards, et al., 2012).

A partir de esta observación, el educador puede detectar los intereses de los niños y niñas, y a partir de ello, desarrollar las experiencias y proyectos que han de articular los procesos pedagógicos. En cuanto al trabajo en base a proyectos en el modelo Reggio Emilia, Rinaldi (2005, p.19) nos explica:

El trabajo por proyectos crece en muchas direcciones, sin una progresión predefinida, sin resultados decididos antes de que empiece el trayecto. Significa ser sensibles a los resultados imprevisibles de la investigación y estudio de los niños. Entonces, el curso de un proyecto puede ser corto, mediano o largo, continuo o discontinuo, y está siempre abierto a modificaciones y cambios de dirección (en Edwards, Gandini \& Forman, 2012, p. 111).

Al conocer al grupo de niños y niñas en sus intereses, es importante tener presente lo que sabemos sobre su aprendizaje y desarrollo. Por lo tanto, toma relevancia también el cuidado que 
debe darse a la estética, siendo que la búsqueda de la belleza es una necesidad intrínseca de nuestra especie, y que demostramos desde nuestros primeros años de vida. Esta dimensión estética es definida por Vecchi (2006) como "una relación empática y sensible con el entorno, un hilo que conecta y ata las cosas entre si [...]; elecciones en las que se percibe armonía, cuidado, placer para la mente y los sentidos" (p. 15).

Desde muy tempranas edades aparecen formas de buscar estas relaciones, la importancia del mundo sensorial es enormemente visible en las formas de exploración de los niños. La sensación es el proceso mediante el cual los órganos de los sentidos responden a los estímulos que reciben, por lo tanto, las sensaciones son el punto de partida del conocimiento (Sisalima \& Pizarro, 2013); mientras que la percepción se entiende como el proceso físico y emocional de interpretar, analizar e integrar lo previamente sentido (Bainbridge-Cohen, 1993; Sisalima \& Pizarro, 2013).

Esta relación del mundo sensorio-perceptivo es aquello que nos permite acercarnos a lo que Vea Vecchi (2013) plantea como dimensión estética de la belleza, y por tanto, de la relación que establecemos con el mundo como seres humanos:

Quizá una cuestión importante que aceptar sea la búsqueda de la belleza forma parte de nuestra especie de modo profundo y natural y que constituye un elemento importante de nuestra humanidad, una necesidad primaria. [...] Esta forma de inspiración se encuentra en todos los pueblos y culturas, pasados y presentes: Hacer estético, entendido y experimentado como un filtro para interpretar el mundo, una actitud ética, una forma de pensar que requiere cuidado, gracia, atención, sutileza y humor, un enfoque mental que va más allá de la simple apariencia de las cosas para destacar aspectos y cualidades inesperados. (p. 64)

En esta integración del ser con la percepción, aparece en el mundo infantil la atracción innata hacia la narración oral, como un medio de interacción y conexión con otros. Su belleza se transforma en una nueva dimensión estética del mundo, en donde la cultura y la comunidad 
"Narraciones cotidianas", una experiencia de audiencia con niños/as de 1 a 2 años

aparecen en interrelación. Schenck (2014), plantea este aspecto como una atracción que va más allá de la comprensión puramente semántica de lo narrado, es "un algo que tal vez se encuentre enraizado con ancestrales búsquedas de códigos de comunicación que puedan ser compartidos en una «aceptación mutua»" (Maturana, 1997, p. 32), ante lo cual una de las formas en que la literatura se manifiesta en plenitud es el teatro. De acuerdo a Bonilla (2014), "en el teatro hay lenguaje, forma, color, movimiento y contenido" (p. 93), por lo que permite una versatilidad al momento de pensar las formas de incorporar la narración desde la perspectiva de lo contemporáneo y sobre todo en relación al mundo infantil.

Planteamos en torno a esta construcción de narrativas, aquello que consideramos como apreciación estética en los primeros años, vinculando elementos desde la apreciación en otras etapas del ciclo vital, así como profundas conexiones desde lo que es propio de las etapas tempranas. Podemos pensar que se trata de percibir con sensibilidad, viviendo el complejo mundo de las emociones, interpretando la información sensorial que nos entrega el entorno, conectándonos con nuestro ser y su memoria, generando así el conocimiento y una opinión reflejada desde nuestra corporalidad.

\section{La experiencia: de la teoría a la práctica}

La experiencia se realiza en Küpülwe, jardín infantil privado, inserto en un barrio residencial en Santiago de Chile. El Proyecto Educativo Institucional del centro está inspirado en la filosofía Reggio Emilia, donde el pilar fundamental del proceso pedagógico son los proyectos establecidos desde los intereses de los niños de cada sala. El período de ejecución de la misma es el segundo semestre del año 2018, en Sala Agua, con 13 niños entre los 1 y 2 años.

La experiencia se desarrolló a nivel transversal en el aula, implementando diversas experiencias de narración, utilizando estrategias orales, corporales, sonoras y visuales. 
Desde abril del 2018, se comenzaron a gestar proyectos de sala en diferentes temáticas y a través de instalaciones estéticas. La instalación como práctica artística, posibilita la implicación corporal de los niños facilitando la construcción de la identidad y la conquista de un espacio. El objetivo es que el niño se convierta en un "explorador activo", experimentando y vivenciando lo espacios y materiales ofrecidos (Moya, M. 2017). Con base en ello, se observaron los intereses genuinos de los niños durante los diferentes periodos de indagación en el juego libre durante la jornada.

Hacia fines de agosto del mismo año, se detecta un interés que convoca al grupo en torno a un espacio permanente dentro del aula, destinado a los libros y narraciones, así como el interés por permanecer en atención plena y juego sin intervención del adulto para su motivación. Esto se hizo visible en acciones como mantenerse por largos momentos hojeando de forma secuencial las ilustraciones, pidiendo lecturas dialogadas a los adultos en sala y comentar con otros, acciones específicas de ciertas ilustraciones. También se visibilizó en el momento del círculo, en el cual se cantaban canciones con historias acompañadas de mímicas, escogidas por los mismos niños cada día. Al cabo de un tiempo, las canciones comenzaron a ser cada vez de mayor complejidad y profundidad, y los niños empezaron a añadirles frases que las finalizaban o bien que interpretaban algunas de ellas.

- Un ejemplo de esto es cuando Mariel (nombre ficticio) incorpora una nueva frase al círculo: Todos (Cantando): “A mi mono le gusta la lechuga, planchadita sin ni una sola arruga, se la come con sal y con limón, muy contento sentado en su sillón”. Mariel: “Es muy grande su sillón".

Desde este interés aparece un proyecto nuevo y amplio, en relación, con la necesidad de involucrar a los niños con propuestas que tuvieran un sentido cultural en torno a lo propio, nacional e incluso latinoamericano: la valoración de la narración oral como medio literario y las complejidades que aporta el mundo artístico en su interpretación y vivencia. 
"Narraciones cotidianas", una experiencia de audiencia con niños/as de 1 a 2 años

Se escogió la formación de audiencia infantil como gran teoría base asociada, entendiéndola desde la lógica de apreciación estética en los primeros años, y dándole un vuelco a lo que significaba la conexión vincular que los niños buscaban con sus acciones. A lo largo del semestre, se crean entonces “obras” (entendidas más bien como una mezcla de performance, instalaciones de arte contemporáneo y teatro, las cuales invitan a vivirlas desde un punto de vista de audiencia participante) desde la belleza de lo simple, el arte y la sensorialidad, permitiendo a los niños envolverse en el mundo de la literatura, integrando las distintas dimensiones de las artes, permitiendo involucrarse profundamente con la apreciación como modo de investigación.

De esta manera, se pensaron una amplia variedad de herramientas y estrategias para utilizar en las "obras". Enfocándose en aspectos visuales, sonoros, corporales, o una mezcla de varios, se logró generar una estética propia de cada una de las narraciones escogidas para su representación. Estas fueron interrelacionándose con los proyectos que sucedían paralelamente en el aula, transformándose en un proyecto transversal, en vinculación con diferentes áreas de interés y los fenómenos del mundo que los niños estaban investigando.

En cada una de las "obras", se generó una ritualidad que antecede y prosigue tanto a la "obra" como a la intervención.

- Primero, utilizando siempre la misma canción inicial y distribución espacial, se dispone a los niños a la escucha atenta, al igual que un espectador se prepara cuando se apagan las luces en el teatro.

- Segundo, se realiza el desarrollo de la "obra", narrada y propuesta desde una lógica interdimensional, en donde la palabra emerge en cadencias y tonalidades diferentes, en conjunción a los distintos elementos visuales, sonoros y corporales de la propuesta.

- Tercero, a través de un gesto o una palabra, se invita a los niños a entrar al espacio escenográfico e intervenir sobre los materiales dispuestos, permitiendo la transformación de la realidad a partir de la "obra" por parte de los niños. 


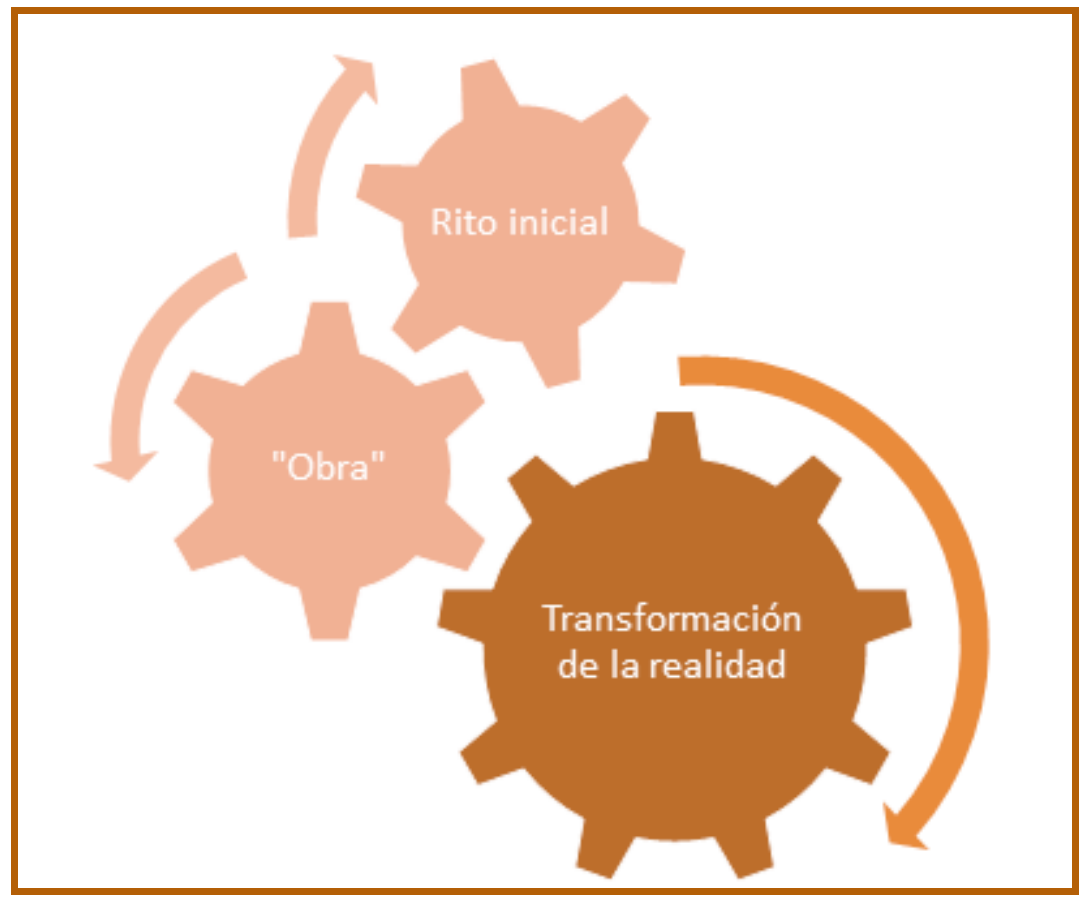

Fig. 1. Ritualidad en torno a la provocación.

A continuación, se detalla la serie de "obras" y narraciones que se realizaron a lo largo de todo el semestre:

a. El baño de Elmer: "Obra" visual del libro "El baño de Elmer" de David Mckee. Utilizando una esponja pintada con témpera para representar al personaje principal, papel, agua y jabón, se realiza la narración. La cotidianeidad de la historia y el asombro en torno a este juego sensorial con la espuma y el color, permitió a los niños generar un vínculo emocional desde el cual vivenciaron el baño del elefante Elmer.

b. La tierra: Una "obra" corporal creada también como parte del proyecto de ancestralidad que se desarrollaba en paralelo, donde el poema "La tierra" de Gabriela Mistral fue la inspiración que guio el proceso creativo y una profunda investigación teórica y sonora en 
torno a los latidos ancestrales y el significado cultural del sonido del tambor. Los elementos asociados a la tierra simbolizados en la lana, el papel, la arena y las percusiones, abrieron posibilidades a los niños de hacerse parte de la "obra", transformando lo que antes era parte de la instalación y creando algo completamente nuevo y único a su vivencia.

c. Cosita linda: Un teatro de sombras a partir del libro "Cosita linda" de Anthony Browne, creado también para el proyecto de la luz que se desarrollaba en paralelo. En esta "obra", el montaje fue la clave: adentrarse a un espacio que durante la "obra" estaba oculto, generó una atmósfera emocional en la que el niño se volvió protagonista, interviniendo sobre el material y la luz, provocando un efecto colectivo.

d. Cuenta cuentos: Se invita a una cuenta-cuentos profesional, quien presenta a los niños una de sus historias en la cual una niña del campo era la protagonista. Utilizando un abrigo bordado con el paisaje narrado, el instrumento musical andino "pito de agua", y títeres, transporta a los niños a los diversos escenarios donde se desarrollaba la historia. Los niños se fueron involucrando en la narración e interviniendo los recursos ofrecidos, vinculándose con la narradora y sus recursos materiales.

e. Susurradores: Se construyen susurradores, un artefacto inventado en el 2001 por una compañía francesa llamada Les souffleurs. Son un puente entre el narrador y el "espectador" que escucha, a través del cual se susurran historias, creando un lazo poético e íntimo (Fundación Mustakis, s.f.). En esta ocasión, se decidió narrar las canciones del círculo, historias que los niños ya conocían, generando en ellos una cercanía afectiva en grupos de íntima relación (2 a 3 niños por grupo).

f. Teatro de títeres: Se monta un escenario para interpretar el folclor poético "Estaba la rana cantando debajo del agua". La dinámica establecida, permitió a los niños interactuar de forma activa durante la "obra", y al final, los llevó a crear narraciones a ellos también. 
En el siguiente diagrama, se detalla la forma en cómo se constituye la transversalidad y línea temporal del proyecto de formación de audiencia infantil, interrelacionándose con los demás proyectos de aula, así como en su estructura durante el segundo semestre del 2018:

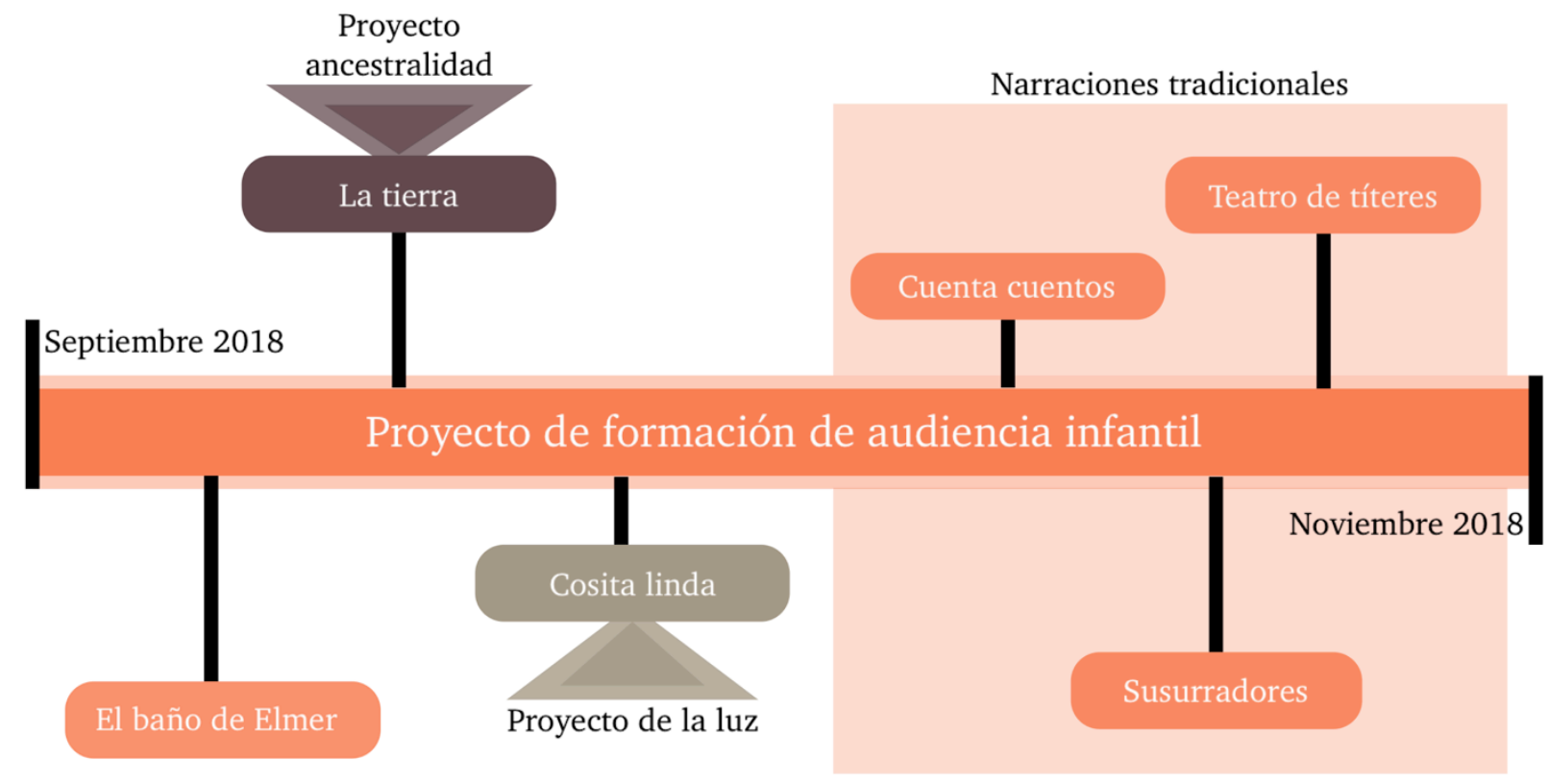

Fig. 2. Línea temporal del proyecto de formación de audiencia infantil.

\section{Resultados y discusión a partir de los procesos del proyecto - Una mirada hacia lo emergente}

Al finalizar las experiencias que componen el proyecto, se realiza una evaluación y análisis de lo realizado y vivido por todos actores que formaron parte de las implementaciones. A partir de esto, surgen los distintos procesos que fueron sucediendo a medida que se desarrollaba el mismo y que, para efectos de comprensión, se han divido en dos: los vividos por las educadoras y los vividos por los niños y niñas (Fig. 3. Procesos vividos durante la implementación del proyecto de formación de audiencia infantil). 


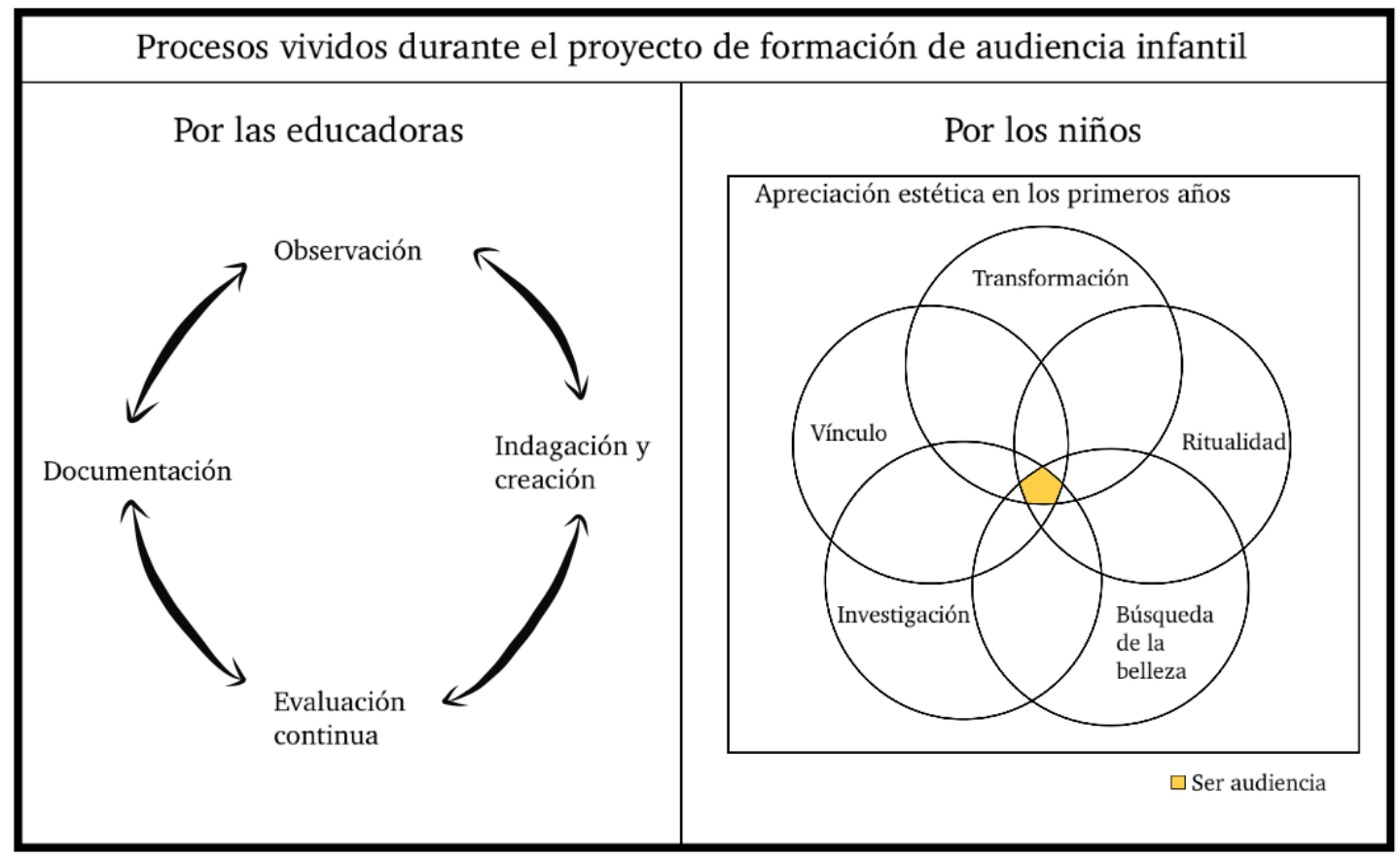

Fig. 3. Procesos vividos durante la implementación del proyecto de formación de audiencia infantil.

\section{A. Los procesos vividos por las educadoras durante la implementación del Proyecto:}

Los procesos vividos por las educadoras se interrelacionan y se dan de forma cíclica a lo largo del proyecto. Estos se detallan a continuación:

\section{- Observación}

A partir de la observación, las educadoras tuvieron la posibilidad de escuchar activamente y conocer a los niños y sus intereses. Se da en un principio para iniciar el proyecto y se extiende a lo largo de cada una de las experiencias, en las que las educadoras observan a los niños, sus reacciones, acciones e intervenciones en cada una de las "obras". Los registros que resultan de esta actividad, son el insumo de conocimiento que permite el desarrollo del resto de los procesos. 


\section{- Indagación y creación}

Se vive este proceso en la creación de cada una de las "obras" presentadas a los niños. Las educadoras se toman la tarea de indagar, investigando conceptos, estrategias, materialidades y literatura - tanto teórica como en experimentación corporal - para entonces crear cada "obra". A lo largo del mismo, se tiene siempre en consideración la necesidad de ofrecer y permitir, a través de las materialidades y narrativas, la aparición de una diversidad de lenguajes y de la sensorialidad como medio para conocer y construir sobre el mundo, a la vez que se tiene especial cuidado de la estética, invitando al deleite frente a la belleza de la "obra" en su completitud.

\section{- Evaluación continua}

Las educadoras con base a las observaciones que realizan en cada intervención, tienen la posibilidad de evaluar de forma continua la experiencia; esto a partir de conversaciones y la reflexión profunda y crítica. ¿Qué cosas salieron bien o no en términos de la planificación e intervención del adulto? ¿Cómo lo vivieron los niños? Qué investigaciones y transformaciones realizaron los niños, interpretando los conocimientos creados y las formas de vincularse con lo propuesto. En base a estas reflexiones, se piensan nuevas formas de crear y hacer para las siguientes experiencias.

\section{- Documentación}

Otro proceso evaluativo que surge, es el de la documentación. A partir de este, las observaciones y evaluaciones continuas se organizan, interpretan y comparan con la teoría. De este proceso, muchas veces resultan documentos enfocados en un aspecto de lo vivido, y en el cual se incluyen fotografías, registros de observación, interpretaciones desde la teoría y proyecciones a futuro a través de preguntas indagatorias que surgen de las interpretaciones de las educadoras, y que buscan darle continuidad y complejidad a la vivencia del niño. 
"Narraciones cotidianas", una experiencia de audiencia con niños/as de 1 a 2 años

\section{B. Procesos vividos por los niños y niñas}

En cuanto a los procesos vividos por los niños y niñas, se han englobado dentro de lo que se considera como apreciación estética para estas edades, y al interrelacionarse, se encuentran todos en la experiencia de "ser audiencia". A continuación, se detallan estos procesos:

\section{- Ritualidad}

Considerando las herramientas con las que los niños se desenvuelven en el mundo cotidiano, la ritualidad aparece en forma de ritos e hitos que denotan rutina y consistencia temporal. Aquello que los niños comienzan a identificar como propio de sus rutinas, y por lo tanto, propio de sus procesos diarios y constituyentes de sus actividades diarias, comienza a anticipar a los niños respecto a lo que está por venir, y así mismo se correlaciona con el acto de participar como una audiencia, tanto en edades tempranas como en edades mayores.

Al encontrarnos frente a un teatro, con sus luces tenues y las cortinas cerradas, o bien, al ver acercarse gente a una aglomeración en la calle hacia una fuente sonora, sabemos que nos encontramos en una nueva disposición: en pos de ser audiencia de aquello que nos permea el mundo escénico. En el caso de los niños, desplazarse hacia un espacio preparado, vivir el rito de inicio, experimentar la apreciación estética de la "obra", para finalmente intervenir y transformar, fue la ritualidad que les permitió anticipar, prever y disfrutar con mayor plenitud aquel espacio de seguridad y de múltiple provocación, dentro de este mundo que ya se volvía habitual para ellos.

\section{- Búsqueda de la belleza}

En el continuo de la percepción del mundo que nos rodea, el ser humano se asombra y confunde con lo que la vida en sí misma ofrece. La mirada a la que nos invita, nos deleita de belleza y de visión estética, en donde las significaciones y vinculaciones emotivas conmueven y dan sentido a aquello que percibimos. El niño no está exento de belleza en su andar y deleite de su mundo circundante, más bien, goza de una innata necesidad de establecerla como parte de su cotidiano. Desde la conexión humana, hasta la transformación de las materialidades, así como la ritualidad y la repetición de acciones (propias de la etapa temprana), han de estar llenas de belleza, donde 
los ritmos y los procesos cognitivos buscan incansablemente elementos de estética en su propia forma. Así es como los niños se acercan a la "obra", dispuestos a encontrar la belleza en aquello que les es propuesto. Luego, son capaces de establecer nuevas formas de interacción profunda con los materiales a partir de sus vivencias personales y a través de una transformación provocadora y profundamente estética.

\section{- Investigación}

Tal como el mundo es bello, es intrigante, y existen miles de preguntas que quisiéramos que fuesen respondidas y es la curiosidad aquel motor para el profundo ahínco indagatorio. Una experiencia llama a vivir concepciones sobre lo que nos rodea, y como seres pensantes, nuestras mentes se activan en busca de preguntas y respuestas del mundo. Existe una profunda relación entre lo que los niños viven, repiten y replican de otros, que nos lleva a imaginar un mundo interior enriquecido en cuestionamientos. Es, en aquel momento en que un niño realiza intencionalmente una acción repetidas veces, cuando está investigando el mundo, tratando de ver qué es aquello que le llama tanto la atención, o bien, cómo es que aquello que ya descubrió, ocurre realmente.

La propuesta de formación de audiencia se involucra con este estado del niño, ya que es la forma más profunda de relacionarse con un escenario crítico y de amplia interpretación como lo son las artes. Es por esto que la filosofía Reggiana acoge en sintonía nuestra propuesta, en la que la investigación de los procesos de los niños y cómo ellos teorizan sobre los sucesos del mundo, traspasa el escenario artístico al proponer, en una narrativa visual, sonora y corporal, aquello que los niños tanto ansían por conocer, replicar y re-significar. La investigación que realizan los niños se vuelve entonces un proceso dual, desde lo artístico-emocional que emerge en la vivencia, y que, a su vez, permite a los niños relacionarse con aquello que les fue dispuesto en la "obra" desde perspectivas indagatorias. 


\section{- Vínculo}

La experiencia significativa solamente existe cuando se vive desde la afectividad. Esta curiosidad tan propia de los niños y tan efímera en el mundo adulto, actúa como motor que nos lleva a la investigación, y es la principal emoción que nos infunde el deseo por conectarnos con todo lo que nos rodea. Así, desde la afectividad, vamos vinculándonos con el mundo circundante. Ese vínculo que se genera, es lo que transforma cualquier experiencia en una experiencia significativa, dejando una huella en el ser y encendiendo, a través del asombro, el goce y la emocionalidad, el afán por seguir generando lazos con otros y con nuestro entorno.

$\mathrm{Al}$ enfrentarse a las propuestas del proyecto, el niño vive un proceso de conexión con su propio ser, en el que evoca su memoria y experiencias vividas. Desde este lugar, puede entonces vincularse con las narrativas desde la visualidad, sonoridad y corporalidad, para así generar conocimientos significativos, establecer relaciones entre su vida y la "obra", así como expresar sus opiniones mediante diversos lenguajes.

\section{- Transformación}

A su vez, desde su mirada subjetiva, invitan a los demás a sumarse a este mundo de descubrimientos sobre sus propias capacidades de ejercer cambios en su entorno cercano. Al observar la obra, vivenciarla y plasmar en concreto mediante la intervención, todo su imaginario va transformando el entorno inmediato. Mediante la investigación, que se refleja en la acción, en el ensayo y error, y en la vinculación al encontrarse con un otro en un espacio relacional, se comparten momentos o experiencias donde algunas ideas se aúnan, donde risas, miradas y movimientos se vuelven un camino y transforman juntos la obra.

Transformar entonces, se traduce en un lenguaje sutil y sublime, que nace del mundo interior de cada niño, y que, desde su pensamiento individual, se conjuga con otros pensamientos, para darle un giro a la propuesta. Re-creando, re-pensando, re-formulando, re-haciendo la "obra" que se presenta ante su mundo. Tomo lo que me mueve de lo observado y vivenciado, lo llevo a la acción para armarlo nuevamente desde las ideas de mi propio mundo, y así me encuentro con otro/a quien comparte conmigo estos intereses y juntos, transformamos el espacio. 


\section{Conclusiones}

El proyecto de formación de audiencia infantil partió del niño como centro de toda práctica pedagógica; a partir de esta premisa, a través de la observación y escucha activa, se buscó dar respuesta a una necesidad e interés que demostraron los niños del grupo por las narraciones orales y su relación con la belleza de la conexión humana. Desde la filosofía Reggiana, que sustenta la práctica pedagógica del centro educativo en el que se desarrolla el proyecto, se crean las diversas "obras" que responden a la formación de audiencia en sí misma, a los distintos proyectos que se vivían en paralelo en la sala, y a narraciones de la tradición oral.

Al finalizar las intervenciones, y como resultado de este proyecto, surgió la visibilización de diversos procesos que vivieron las educadoras y los niños. La observación, la indagación y creación, la evaluación continua y la documentación, fueron los procesos vividos por las educadoras. Englobados dentro de la apreciación estética en los primeros años, la ritualidad, la búsqueda de la belleza, la investigación, el vínculo y la transformación, fueron los procesos que vivieron los niños, los cuales al interrelacionarse se encontraban en el "ser audiencia".

A partir de este proyecto, se puede proyectar la creación de una propuesta de formación de audiencia infantil para los primeros años que incorpore y detalle más en profundidad todos los procesos que se vivieron, elaborando en nuevas prácticas, grupos y edades tempranas, que pudiesen continuar esta búsqueda de nuevas perspectivas para ahondar en las capacidades de los niños para vincularse con las propuestas educativas, así como sus posibilidades de acción sobre lo que nos parece tan lejano como la cultura y la comunidad. 
"Narraciones cotidianas", una experiencia de audiencia con niños/as de 1 a 2 años

\section{$\underline{\text { Referencias }}$}

Bainbridge-Cohen, B. (1993). Sensing, Feeling and Action. The Experiential Anatomy of BodyMind Centering. Northampton: Contact Editions.

Bonilla Martínez, M. (2014). El teatro en la educación y su importancia. El teatro va a la escuela. Organización de Estados Iberoamericanos para la Educación, la Ciencia y la Cultura. Pp. 79-93.

Edwards, C. P., Gandini, L., \& Forman, G. E. (2012). The hundred languages of children: the Reggio Emilia experience in transformation (3ra ed.). Santa Barbara, California: Praeger.

Fundación Mustakis. (S.f.) Los susurradores y los PUPs. Escuela de Cuenta Cuentos.

Moya, M. (2017). Universidad de Valladolid. Segovia. Recuperado de: https:/uvadoc.uva.es/bitstream/handle/10324/24655/TFG

B.1081.pdf\%3Bjsessionid=AF54487EAFE9446ABB26F3D9AE295965?sequence=

Red Territorial de Educación Infantil de Cataluña. (2011). Documentar la vida de los niños y las niñas en la escuela (1 era ed.). Barcelona: Octaedro.

Schenck, L. (2014). La literatura en la primera infancia. Arte, Educación y Primera Infancia: Sentidos y Experiencias. Organización de Estados Iberoamericanos para la Educación, la Ciencia y la Cultura. Pp. 29-43.

Sisalima Pizarro, B. K., \& Vanegas Vintimilla, M. F. (2013). Importancia del desarrollo sensorial en el aprendizaje del niño. Repositorio Institucional Universidad de Cuenca, Cuenca.

Vecchi, V. (2006). Prólogo: Estética y aprendizaje. En La estética en el pensamiento y obra pedagógica de Loris Malaguzzi. Barcelona: Octaedro. Pp. 15-25. 
María Gabriela Carrizo Méndez,

Stephanía Cifuentes Oyarzún, Daniela Faúndez Silva

Vecchi, V. (2013). Arte y Creatividad en Reggio Emilia. El papel de los talleres y sus posibilidades en educación infantil. Madrid: Ediciones Morata, S.L. 